\title{
MODULUS ON GRAPHS AS A GENERALIZATION OF STANDARD GRAPH THEORETIC QUANTITIES
}

\author{
NATHAN ALBIN, MEGAN BRUNNER, ROBERTO PEREZ, PIETRO POGGI-CORRADINI, \\ AND NATALIE WIENS
}

\begin{abstract}
This paper presents new results for the modulus of families of walks on a graph - a discrete analog of the modulus of curve families due to Beurling and Ahlfors. Particular attention is paid to the dependence of the modulus on its parameters. Modulus is shown to generalize (and interpolate among) three important quantities in graph theory: shortest path, effective resistance, and max-flow or min-cut.
\end{abstract}

\section{INTRODUCTION}

Beginning with an example, consider the simple, undirected graph shown in Figure 1. Such a graph may be used to model, for example, a contact network for use in predicting the spread of disease, where each vertex indicates an individual in a population and each edge indicates that the two corresponding individuals have direct contact with each other. The disease is then assumed to spread throughout the network according to some stochastic process. An important question in such a model is the following. How easily can individual $s$ transmit the disease to individual $t$ ? In a sense, we are asking for a distance-like measure between $s$ and $t$.

One obvious approach would be to define distance in the classical graph distance sense: the distance between $s$ and $t$ is the length of the shortest path connecting them. In this case, $s$ and $t$ would be at distance 1 from each other. For this particular application, however, this definition seems inappropriate. In graph distance, $s$ is equidistant from $u$ and $t$. However, any reasonable disease model would indicate that a disease originating in $s$ is more likely to spread to $t$ than to $u$; there are more avenues for transmission from $s$ to $t$.

Taking this shortcoming into account, we might try to define proximity through the number of distinct transmission pathways. That is, we could count the maximum number of pairwise edge-disjoint paths from $s$ to $t$ : 3 in this case. By Menger's theorem, this is equivalent to the minimum number of edges that can be removed from the graph in order to separate $s$ from $t$. This min-cut sense of proximity addresses the earlier concern with graph distance, since $s$ is now three times closer

Received by the editors June 1, 2015 and, in revised form, October 30, 2015.

2010 Mathematics Subject Classification. Primary 90C35.

Key words and phrases. Modulus of families of walks, effective resistance, shortest path, maxflow, min-cut.

This material is based upon work supported by the National Science Foundation under Grant No. 126287 (Albin, Brunner, Perez, Wiens), through Kansas State University's 2014 Summer Undergraduate Mathematics Research program, and under Grant Nos. 1201427 (Poggi-Corradini) and 1515810 (Albin). 


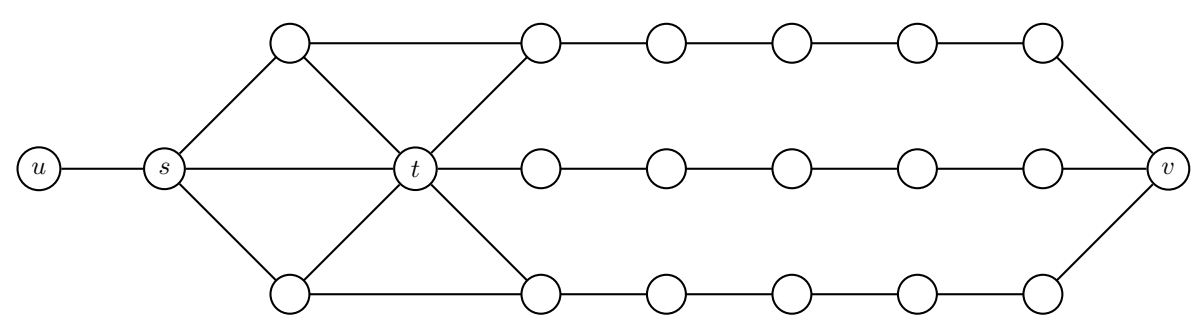

Figure 1. Example graph for Section 1.

to $t$ than to $u$. However, this measure is also lacking; $t$ is now equally close to both $s$ and $v$, which again seems counterintuitive for the application.

A more recent description of distance that bridges the gap between these two extremes is the resistance distance; see for instance [12. The resistance distance between $s$ and $t$ is defined as the effective resistance between the corresponding nodes in an electrical resistor network wherein the vertices of the graph correspond to junctions and the edges to resistors of unit resistance. In resistance distance, $s$ and $t$ are 0.454 units apart, $s$ and $u$ are 1 unit apart, and $t$ and $v$ are 1.918 units apart.

Although resistance distance does give a more satisfying answer for the contact network application, a number of interesting questions remain. For example, although the shortest path and min-cut distances have straightforward extensions to directed graphs, effective resistance does not. So, what is the natural analog of resistance distance for directed graphs? Or consider the following related type of question for the graph in Figure 1. It is clear that $s$ plays a crucial role in the transmission of disease between $u$ and $t$. But, how might we quantify the role that $t$ plays in transmission between $s$ and $v$ ? In other words, how rich is the set of pathways connecting $s$ to $v$ through $t$ ? And how does this compare to the set of all pathways connecting $s$ to $v$ ? Or for that matter, given any interesting collection of walks on the graph, how does one quantify the richness of the collection?

A potential answer to these questions comes in the form of the modulus - a discrete analog of the modulus of curve families dating at least as far back as Beurling [4 and Ahlfors [1. Although discrete versions of the modulus have been introduced before, the goal of the present work is to make explicit the connection to more traditional graph theoretic concepts:

- We treat the modulus in a general framework, accommodating directed or undirected graphs, with or without edge weights (Section 2). Although this is not entirely new (see Section 7 for a description of related work), we do adopt a particular viewpoint that lends itself well to graph theoretic interpretations of the modulus.

- After framing the modulus as a convex optimization problem, we derive and interpret a dual formulation of the modulus (Section 3). This is similar to the duality of the max-flow and min-cut problems, but much more general than has been done before.

- Using the primal-dual formulation, we establish three connections to graph theory, namely that computing the modulus in certain circumstances is equivalent to computing either the graph distance, min-cut distance, or resistance distance (Section 4). Moreover, we prove that the modulus (which 
depends on a continuous parameter $p$ ) effectively interpolates among these three concepts (Section [5).

- In Section 2, the modulus is defined through real-valued densities on the edge set of the graph. We show that these densities generalize the concept of potential drop across resistors in the computation of resistance distance (see Theorem 4.2) and analyze the behavior of these densities (Section 6).

\section{The Modulus}

2.1. Graphs, walks, and families of walks. In what follows, $G$ is a graph with vertex set $V$ and edge set $E$. The sets of vertices and edges are assumed to be finite, with $n=|V|$ vertices and $m=|E|$ edges. The graph may be directed or undirected. In the former case, $E$ is represented as a subset of ordered pairs of vertices, in the latter as a subset of unordered pairs. We adopt the notation $e=(x, y)$ to represent an edge in either case, with the understanding that $(x, y)=(y, x)$ in the undirected case. For any function $f: E \rightarrow \mathbb{R}$ and any edge $e=(x, y)$ we will use the notation $f(e)$ and $f(x, y)$ interchangeably.

The discussion in this paper is restricted to simple graphs, though there are natural extensions to multigraphs and to other more general objects. In general, $G$ is assumed to be weighted, with weight function $\sigma: E \rightarrow(0, \infty)$. A graph is called unweighted if $\sigma \equiv 1$. For the purposes of this paper, then, a graph is defined through the triple $G=(V, E, \sigma)$.

Using the standard terminology, a walk on $G$ is represented as

- a finite string of vertices $v_{1} v_{2} v_{3} \ldots v_{r+1}$ in $V$, with the property that $\left(v_{i}, v_{i+1}\right) \in E$ for $i=1,2, \ldots, r$ or, equivalently,

- the corresponding string of edges $e_{1} e_{2} \ldots e_{r}$, where $e_{i}=\left(v_{i}, v_{i+1}\right)$ for $i=$ $1,2, \ldots, r$.

In either case, we require that $r \geq 1$, so that a walk is considered to traverse at least one edge in the graph.

To each walk $\gamma=e_{1} e_{2} \ldots e_{r}$ is associated the graph length, $\ell(\gamma):=r$. That is, $\ell(\gamma)$ counts the number of "hops" made in traversing $\gamma$. More generally, given any edge density $\rho: E \rightarrow \mathbb{R}$, the $\rho$-length of $\gamma, \ell_{\rho}(\gamma)$, is defined as

$$
\ell_{\rho}(\gamma):=\sum_{i=1}^{r} \rho\left(e_{i}\right) .
$$

The graph length of $\gamma$ coincides with the $\rho$-length for the particular choice $\rho \equiv 1$.

Throughout this paper, $\Gamma$ represents an arbitrary nonempty family of walks on $G$. A particularly useful class of walk family is the connecting family, denoted $\Gamma(s, t)$, of walks originating at a vertex $s \in V$ and terminating at a distinct vertex $t \in V \backslash\{s\}$.

2.2. Densities and admissibility. Given a walk family $\Gamma$ and an edge density $\rho: E \rightarrow \mathbb{R}$, the graph length and $\rho$-length of $\Gamma$ are defined as

$$
\ell(\Gamma):=\inf _{\gamma \in \Gamma} \ell(\gamma) \quad \text { and } \quad \ell_{\rho}(\Gamma):=\inf _{\gamma \in \Gamma} \ell_{\rho}(\gamma)
$$

respectively.

The family $\Gamma$ determines three sets of densities called the admissible set $A(\Gamma)$, the relaxed admissible set $A^{\prime}(\Gamma)$, and the restricted admissible set $A^{*}(\Gamma)$ defined as 
follows:

$$
\begin{aligned}
A^{\prime}(\Gamma) & :=\left\{\rho: E \rightarrow \mathbb{R}: \ell_{\rho}(\Gamma) \geq 1\right\}, \\
A(\Gamma) & :=\left\{\rho: E \rightarrow \mathbb{R}: \ell_{\rho}(\Gamma) \geq 1, \quad 0 \leq \rho\right\}, \\
A^{*}(\Gamma) & :=\left\{\rho: E \rightarrow \mathbb{R}: \ell_{\rho}(\Gamma) \geq 1, \quad 0 \leq \rho \leq 1\right\} .
\end{aligned}
$$

Note that these definitions are made in the order of decreasing size: $A^{*}(\Gamma) \subset$ $A(\Gamma) \subset A^{\prime}(\Gamma)$. The reason for defining three different admissibility sets will be apparent by Remark 2.1

2.3. Energy and modulus. Given a real parameter $p \geq 1$ or $p=\infty$, the $p$-energy of a density $\rho$ is

$$
\mathcal{E}_{p}(\rho):= \begin{cases}\sum_{e \in E} \sigma(e)|\rho(e)|^{p} & \text { if } 1 \leq p<\infty, \\ \max _{e \in E}|\rho(e)| & \text { if } p=\infty .\end{cases}
$$

The definition for $\mathcal{E}_{\infty}$ is consistent in the sense that

$$
\forall \rho: E \rightarrow \mathbb{R} \quad \lim _{p \rightarrow \infty} \mathcal{E}_{p}(\rho)^{1 / p}=\mathcal{E}_{\infty}(\rho) .
$$

For $1 \leq p \leq \infty$, the $p$-modulus of $\Gamma$ is defined as

$$
\operatorname{Mod}_{p}(\Gamma):=\inf _{\rho \in A(\Gamma)} \mathcal{E}_{p}(\rho) .
$$

(The modulus of an empty family $\operatorname{Mod}_{p}(\varnothing)$ is defined to be zero, since the choice $\rho \equiv 0$ is trivially admissible.)

2.4. Extremal densities. An edge density $\rho \in A(\Gamma)$ is called extremal for a given family $\Gamma$ and a given $p$ if

$$
\operatorname{Mod}_{p}(\Gamma)=\mathcal{E}_{p}(\rho)
$$

The notation $\rho^{*}$ or $\rho_{p}$ is frequently used to denote an extremal density, with the latter used in order to make the dependence on $p$ explicit.

Lemma 2.1. Given $\Gamma$ and $1 \leq p \leq \infty$, there exists an extremal density $\rho^{*}$. If $1<p<\infty$, then $\rho^{*}$ is unique.

Proof. For any $1 \leq p<\infty$, the function $\rho \mapsto \mathcal{E}_{p}(\rho)^{1 / p}$ is a norm on the $m$ dimensional space of edge densities, as is the function $\rho \mapsto \mathcal{E}_{\infty}(\rho)$. Moreover, the set $A(\Gamma)$ is closed and convex. Thus, the modulus problem can be restated as the problem of finding a point in a closed convex subset of $\mathbb{R}^{m}$ that is closest to the origin in a particular norm. Such a point always exists, and is unique provided the norm is strictly convex, as it is in the cases $1<p<\infty$.

The following observation, which shows that the nonnegativity constraint on $\rho$ need not be explicitly enforced, will prove useful in Section 3 .

Lemma 2.2. Let $\Gamma$ and $1 \leq p \leq \infty$ be given. Then

$$
\operatorname{Mod}_{p}(\Gamma)=\inf _{\rho \in A^{\prime}(\Gamma)} \mathcal{E}_{p}(\rho) .
$$

An extremal $\rho^{*} \in A^{\prime}(\Gamma)$ always exists for the relaxed problem, and is unique if $1<p<\infty$. Moreover, for $1 \leq p<\infty$ any such extremal $\rho^{*}$ satisfies the bounds $0 \leq \rho^{*} \leq 1$, and thus $\rho^{*} \in A^{*}(\Gamma)$. When $p=\infty$ there exists at least one extremal $\rho^{*} \in A^{*}(\Gamma)$. 


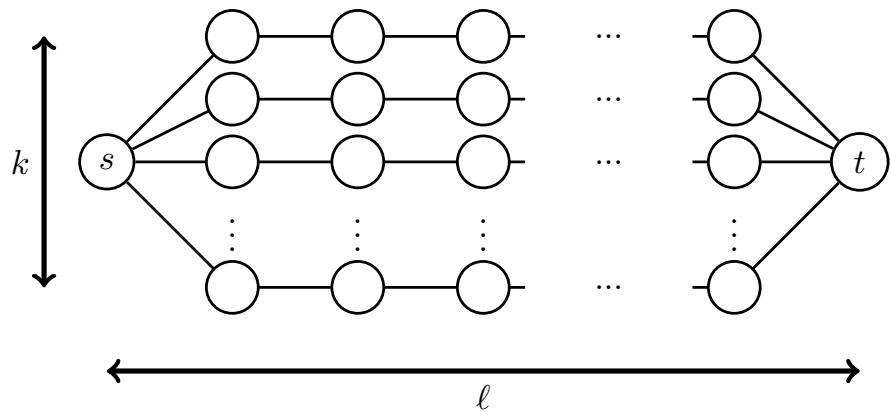

FIGURE 2. A graph consisting of $k$ simple paths with $\ell$ hops each, connecting $s$ to $t$.

Proof. Existence and uniqueness (for $1<p<\infty$ ) of a minimizer for the infimum in Equation (2) are proved as in Lemma 2.1

Now, suppose $\rho \in A^{\prime}(\Gamma)$ takes a negative value on some edge $e^{\prime} \in E$, and define $\rho^{+}$as $\rho^{+}(e)=\max \{\rho(e), 0\}$. Since $\ell_{\rho^{+}}(\gamma) \geq \ell_{\rho}(\gamma) \geq 1$ for any $\gamma \in \Gamma, \rho^{+}$is also admissible. Moreover, on every edge $e \in E,\left|\rho^{+}(e)\right| \leq|\rho(e)|$, and $\left|\rho^{+}\left(e^{\prime}\right)\right|=0<$ $\left|\rho\left(e^{\prime}\right)\right|$. For $1 \leq p<\infty$, this implies $\mathcal{E}_{p}\left(\rho^{+}\right)<\mathcal{E}_{p}(\rho)$, showing that any minimizing $\rho^{*}$ is in $A(\Gamma)$. For the $p=\infty$ case, the inequality $\mathcal{E}_{\infty}\left(\rho^{+}\right) \leq \mathcal{E}_{\infty}(\rho)$ holds, but need not be strict. However, a minimizing $\rho^{*}$ can always be found in $A(\Gamma)$.

Next, suppose $\rho \in A(\Gamma)$ and that $\rho\left(e^{\prime}\right)>1$ on some edge $e^{\prime} \in E$. Define $\rho^{\prime}(e)=\min \{\rho(e), 1\}$. For any $\gamma \in \Gamma$, either $\ell_{\rho^{\prime}}(\gamma)=\ell_{\rho}(\gamma) \geq 1$, or $\gamma$ traverses an edge where $\rho^{\prime}$ takes the value 1 . In the latter case, the inequality $\ell_{\rho^{\prime}}(\gamma) \geq 1$ is trivial, so $\rho^{\prime} \in A(\Gamma)$. Since $\rho^{\prime}(e) \leq \rho(e)$ on all edges, and since the inequality is strict on at least one edge, we have $\mathcal{E}_{p}\left(\rho^{\prime}\right)<\mathcal{E}_{p}(\rho)$ (for $1 \leq p \leq \infty$ ). This proves that $\rho^{*} \leq 1$.

Remark 2.1. A consequence of Lemma 2.2 is that the admissibility set $A(\Gamma)$ in the definition of modulus can be replaced with either a more relaxed set, $A^{\prime}(\Gamma)$, or a more restricted set, $A^{*}(\Gamma)$, without changing the outcome. That is,

$$
\operatorname{Mod}_{p}(\Gamma):=\inf _{\rho \in A(\Gamma)} \mathcal{E}_{p}(\rho)=\inf _{\rho \in A^{\prime}(\Gamma)} \mathcal{E}_{p}(\rho)=\inf _{\rho \in A^{*}(\Gamma)} \mathcal{E}_{p}(\rho) \text { for all } 1 \leq p \leq \infty
$$

A convenient choice of admissibility set can simplify proofs in certain circumstances. For example, when deriving the Lagrange dual problem in the case $1<p<\infty$ (Section 3) it is better to use the most relaxed set $A^{\prime}(\Gamma)$. The proof of Theorem 3.1 utilizes the lower bound in $A(\Gamma)$, while the bounds of Section 5 make use of both upper and lower bounds in $A^{*}(\Gamma)$.

2.5. An instructive example. As described in the introduction, the $p$-modulus is a generalization of three fundamental quantities in graph theory: shortest path length, effective conductance, and min-cut. Before proving these facts, it is useful to explore an example. Consider the (unweighted and undirected) graph shown in Figure 2 comprising $k$ parallel simple paths of $\ell$ hops connecting node $s$ to node $t$ - with $\Gamma=\Gamma(s, t)$, the connecting family of walks defined at the end of Section 2.1. 
It is a straightforward exercise to show that the choice $\rho^{*} \equiv \frac{1}{\ell}$ is extremal for all $1 \leq p \leq \infty$. Thus, for $1 \leq p<\infty$, the modulus of $\Gamma$ is

$$
\operatorname{Mod}_{p}(\Gamma)=\mathcal{E}_{p}\left(\rho^{*}\right)=k \ell\left(\frac{1}{\ell}\right)^{p}=\frac{k}{\ell^{p-1}},
$$

and for $p=\infty$,

$$
\operatorname{Mod}_{\infty}(\Gamma)=\mathcal{E}_{\infty}\left(\rho^{*}\right)=\frac{1}{\ell}
$$

There are several interesting observations to be made. First, as a function of $p$, the modulus is continuous, monotone decreasing, and decays to zero like $\ell^{-p}$ as $p \rightarrow \infty$ if $\ell>1$. All three of these properties are generic, as shown in Section 5 ,

Moreover, for $1<p<\infty$, the modulus depends on both the number of distinct walks, $k$, and on the length of these walks, $\ell$. The modulus is large for $\Gamma$ containing many short walks, and small for $\Gamma$ containing few long walks. For $p$ near 1 , the modulus is much more sensitive to changes in $k$ than to changes in $\ell$, while, for $p$ very large, the modulus is much more sensitive to changes in $\ell$. In the extreme case $p=1$, the modulus loses all dependence on $\ell$ : a large family of walks has large modulus regardless of their lengths. On the other hand, when $p=\infty$ the modulus loses all dependence on $k$ : a family's modulus depends only on the length of the shortest walk and not at all on the number of walks. The case $p=2$ is also interesting. In this case, the modulus is $k / \ell$, which is the effective conductance of the graph viewed as a resistor network between $s$ and $t$ with unit resistors placed on each edge.

Again, these are general properties. Section 4 proves that, in a specific sense, the 1 -modulus is a generalization of the min-cut problem, that the $\infty$-modulus is always equal to the reciprocal of the length of the shortest walk, and that the 2modulus is a generalization of effective conductance. The results of Section 5 , then, show that $p$ can be thought of as a tuning parameter, which adjusts the balance of importance of "many walks" with "short walks" in the modulus.

\section{LAGRANGE DUAL FORMULATION}

The $p$-modulus problem is a convex optimization problem: it involves minimizing the convex function $\mathcal{E}_{p}$ over the convex set $A(\Gamma)$ defined by a set of linear inequalities. Furthermore, it was shown in [3] that this set of inequalities can be assumed finite; more precisely, the following theorem is true.

Theorem 3.1 ([3]). Let $\Gamma$ be a given family of walks on a graph. There exists a finite subfamily $\Gamma^{*} \subseteq \Gamma$ such that $A\left(\Gamma^{*}\right)=A(\Gamma)$.

Such a finite subfamily is called an essential subfamily for the modulus problem. Ensuring that $\ell_{\rho}(\gamma) \geq 1$ is true for all walks in the essential subfamily is equivalent to ensuring the inequality for the entire family. Thus, even when a described set of walks (e.g., the connecting family $\Gamma(s, t)$ ) contains infinitely many members, it can always be assumed that $\Gamma$ has been replaced by a finite essential subfamily (e.g., the simple paths from $s$ to $t$ when $\Gamma=\Gamma(s, t))$. With this assumption, the modulus problem becomes an ordinary convex program, in the sense of [13, Sec. 28], taking 
the form

$$
\begin{aligned}
\text { minimize } & \mathcal{E}_{p}(\rho) \\
\text { subject to } & \sum_{e \in E} \mathcal{N}(\gamma, e) \rho(e) \geq 1 \quad \forall \gamma \in \Gamma, \\
& \rho(e) \geq 0 \quad \forall e \in E,
\end{aligned}
$$

where $\mathcal{N}(\gamma, e)$ is the $|\Gamma| \times|E|$ matrix whose $(\gamma, e)$ entry contains the number of times walk $\gamma$ traverses edge $e$.

Using standard techniques from convex optimization, we define the Lagrangian for the problem as

$$
L_{p}(\rho, \lambda, \mu):=\mathcal{E}_{p}(\rho)+\sum_{\gamma \in \Gamma} \lambda(\gamma)\left(1-\sum_{e \in E} \mathcal{N}(\gamma, e) \rho(e)\right)-\sum_{e \in E} \mu(e) \rho(e),
$$

where $\lambda: \Gamma \rightarrow[0, \infty)$ and $\mu: E \rightarrow[0, \infty)$ are the Lagrange dual variables associated with the admissibility constraints. The usual weak duality statement takes the following form.

Lemma 3.2. Let $\Gamma$ and $p$ be given. Then

$$
\operatorname{Mod}_{p}(\Gamma)=\inf _{\rho: E \rightarrow \mathbb{R}} \sup _{\substack{\lambda: \Gamma \rightarrow[0, \infty) \\ \mu: E \rightarrow[0, \infty)}} L_{p}(\rho, \lambda) \geq \sup _{\substack{\lambda: \Gamma \rightarrow[0, \infty) \\ \mu: E \rightarrow[0, \infty)}} \inf _{\rho: E \rightarrow \mathbb{R}} L_{p}(\rho, \lambda)=\sup _{\substack{\lambda: \Gamma \rightarrow[0, \infty) \\ \mu: E \rightarrow[0, \infty)}} \mathcal{F}_{p}(\lambda, \mu)
$$

where

$$
\mathcal{F}_{p}(\lambda, \mu):=\inf _{\rho: E \rightarrow \mathbb{R}} L_{p}(\rho, \lambda, \mu) .
$$

Given any $\lambda: \Gamma \rightarrow[0, \infty)$ and any $\mu: E \rightarrow[0, \infty)$, weak duality implies that $\operatorname{Mod}_{p}(\Gamma) \geq \mathcal{F}_{p}(\lambda, \mu)$. The problem of maximizing this lower bound over all nonnegative $\lambda$ and $\mu$ is the Lagrange dual problem to the modulus:

$$
\begin{array}{cl}
\operatorname{maximize} & \mathcal{F}_{p}(\lambda, \mu) \\
\text { subject to } & \lambda(\gamma) \geq 0 \quad \forall \gamma \in \Gamma, \\
& \mu(e) \geq 0 \quad \forall e \in E .
\end{array}
$$

Remark 3.1. Because the inequality constraints in (3) are affine, it follows from Theorem 28.2 of 13 that strong duality holds. That is, there exists a saddle point $\left(\rho^{*}, \lambda^{*}, \mu^{*}\right)$ of the Lagrangian. This is an important property of the modulus problem, especially in the context of numerical approximation, and was explored in a specialized setting in [3]. For the present paper, the weak duality result is usually sufficient.

When working with the dual problem, it is convenient to have a more explicit formulation. We derive such formulations in three different cases: $1<p<\infty$, $p=1$ and $p=\infty$.

3.1. The case $1<p<\infty$. When $1<p<\infty$, Lemma 2.2 implies that the $\rho \geq 0$ constraints need not be explicitly enforced in (3), and can thus be removed. The simplified Lagrangian (omitting the dual variables $\mu$ as they are no longer necessary) takes the form

$$
L_{p}(\rho, \lambda):=\sum_{e \in E} \sigma(e)|\rho(e)|^{p}+\sum_{\gamma \in \Gamma} \lambda(\gamma)\left(1-\sum_{e \in E} \mathcal{N}(\gamma, e) \rho(e)\right)
$$


which is differentiable in each $\rho(e)$, since $p>1$. Thus, the value of the dual energy

$$
\mathcal{F}_{p}(\lambda)=\inf _{\rho: E \rightarrow \mathbb{R}}\left\{\sum_{e \in E} \sigma(e)|\rho(e)|^{p}+\sum_{\gamma \in \Gamma} \lambda(\gamma)\left(1-\sum_{e \in E} \mathcal{N}(\gamma, e) \rho(e)\right)\right\}
$$

can be found by solving $\nabla_{\rho} L_{p}=0$. The optimal density, $\rho_{\lambda}$, for a given $\lambda$ must satisfy the stationarity condition

$$
p \sigma(e) \rho_{\lambda}(e)\left|\rho_{\lambda}(e)\right|^{p-2}-\sum_{\gamma \in \Gamma} \mathcal{N}(\gamma, e) \lambda(\gamma)=0 \quad \forall e \in E .
$$

Since $\lambda \geq 0$ and $\mathcal{N}$ has nonnegative entries, the above equation implies that $\rho_{\lambda} \geq 0$ and so

$$
\rho_{\lambda}(e)=\left(\frac{1}{p \sigma(e)} \sum_{\gamma \in \Gamma} \mathcal{N}(\gamma, e) \lambda(\gamma)\right)^{\frac{1}{p-1}} .
$$

After some simplification, this produces the following formula for $\mathcal{F}_{p}(\lambda)$ :

$$
\mathcal{F}_{p}(\lambda)=L\left(\rho_{\lambda}, \lambda\right)=\sum_{\gamma \in \Gamma} \lambda(\gamma)-(p-1) \sum_{e \in E} \sigma(e)\left(\frac{1}{p \sigma(e)} \sum_{\gamma \in \Gamma} \mathcal{N}(\gamma, e) \lambda(\gamma)\right)^{\frac{p}{p-1}}
$$

We conclude that the Lagrange dual optimization problem can be written as

$$
\begin{aligned}
\text { maximize } & \sum_{\gamma \in \Gamma} \lambda(\gamma)-(p-1) \sum_{e \in E} \sigma(e)\left(\frac{1}{p \sigma(e)} \sum_{\gamma \in \Gamma} \mathcal{N}(\gamma, e) \lambda(\gamma)\right)^{\frac{p}{p-1}} \\
\text { subject to } & \lambda(\gamma) \geq 0 \quad \forall \gamma \in \Gamma .
\end{aligned}
$$

Remark 3.2. Because strong duality holds (see Remark 3.1), solving (6) is equivalent to computing the modulus. This provides an interesting interpretation of the modulus: rather than assigning values to edges (through $\rho$ ), we instead assign values to walks in $\Gamma$ (through $\lambda$ ) in such a way as to maximize the dual energy. Equation (5) provides the formula for determining the extremal density $\rho^{*}$ from an extremal $\lambda^{*}$. On the other hand, the extremal $\lambda^{*}$ are typically not unique.

3.2. The case $p=1$. In the case $p=1$, the general Lagrangian in Equation (4) is not differentiable, so the preceding argument does not apply. This issue can be circumvented by replacing the 1-modulus optimization problem with an equivalent problem:

$$
\begin{aligned}
\operatorname{minimize} & \sum_{e \in E} \sigma(e) \rho(e) \\
\text { subject to } & \sum_{e \in E} \mathcal{N}(\gamma, e) \rho(e) \geq 1 \quad \forall \gamma \in \Gamma, \\
& \rho(e) \geq 0 \quad \forall e \in E .
\end{aligned}
$$

Dropping the absolute values from the energy has no effect on the outcome, since the values of $\rho$ are required to be nonnegative. However, without the absolute values, the energy is now differentiable for general densities $\rho: E \rightarrow \mathbb{R}$. 
Introducing the dual variables $\lambda: \Gamma \rightarrow[0, \infty)$ and $\mu: E \rightarrow[0, \infty)$, the Lagrangian for this problem is

$$
\begin{aligned}
L_{1}(\rho, \lambda, \mu) & :=\sum_{e \in E} \sigma(e) \rho(e)+\sum_{\gamma \in \Gamma} \lambda(\gamma)\left(1-\sum_{e \in E} \mathcal{N}(\gamma, e) \rho(e)\right)-\sum_{e \in E} \mu(e) \rho(e) \\
& =\sum_{\gamma \in \Gamma} \lambda(\gamma)+\sum_{e \in E} \rho(e)\left(\sigma(e)-\sum_{\gamma \in \Gamma} \mathcal{N}(\gamma, e) \lambda(\gamma)-\mu(e)\right) .
\end{aligned}
$$

Since the Lagrangian is affine in $\rho(e)$, it is clear that

$$
\mathcal{F}_{1}(\lambda, \mu):=\inf _{\rho: E \rightarrow \mathbb{R}} L_{1}(\rho, \lambda, \mu)=\left\{\begin{array}{ll}
\sum_{\gamma \in \Gamma} \lambda(\gamma) & \text { if } \sigma(e)-\sum_{\gamma \in \Gamma} \mathcal{N}(\gamma, e) \lambda(\gamma)-\mu(e)=0 \\
-\infty & \text { otherwise. }
\end{array} \quad \forall e \in E,\right.
$$

The dual problem involves maximizing $\mathcal{F}_{1}$ over feasible $\lambda$ and $\mu$, and therefore it will never be advantageous to choose the dual variables such that $\mathcal{F}_{1}(\lambda, \mu)=$ $-\infty$ (assuming this can be avoided). In fact, provided $\lambda$ satisfies the inequalities $\sum_{\gamma \in \Gamma} \mathcal{N}(\gamma, e) \lambda(\gamma) \leq \sigma(e)$ (e.g., $\left.\lambda \equiv 0\right)$, a suitable nonnegative $\mu$ can always be defined to make $\mathcal{F}_{1}(\lambda, \mu)$ finite, whereas, if any of the aforementioned inequalities is violated in the choice of $\lambda$, there will be no nonnegative $\mu$ for which $\mathcal{F}_{1}(\lambda, \mu)$ does not evaluate to $-\infty$.

Thus, the dual problem can be written as follows:

$$
\begin{aligned}
\operatorname{maximize} & \sum_{\gamma \in \Gamma} \lambda(\gamma) \\
\text { subject to } & \sum_{\gamma \in \Gamma} \mathcal{N}(\gamma, e) \lambda(\gamma) \leq \sigma(e) \quad \forall e \in E, \\
& \lambda(\gamma) \geq 0 \quad \forall \gamma \in \Gamma .
\end{aligned}
$$

3.3. The case $p=\infty$. In the $p=\infty$ case, the general Lagrangian is once again not differentiable. In this case, an appropriate equivalent problem is the following:

$$
\begin{aligned}
\text { minimize } & t \\
\text { subject to } & \sum_{e \in E} \mathcal{N}(\gamma, e) \rho(e) \geq 1 \quad \forall \gamma \in \Gamma, \\
& 0 \leq \rho(e) \leq t \quad \forall e \in E
\end{aligned}
$$

The associated Lagrangian takes the form

$$
\begin{aligned}
& L_{\infty}(t, \rho, \lambda, \mu, \eta):=t+\sum_{\gamma \in \Gamma} \lambda(\gamma)\left(1-\sum_{e \in E} \mathcal{N}(\gamma, e) \rho(e)\right) \\
& \quad-\sum_{e \in E} \mu(e) \rho(e)+\sum_{e \in E} \eta(e)(\rho(e)-t) \\
& =t\left(1-\sum_{e \in E} \eta(e)\right)+\sum_{e \in E} \rho(e)\left(-\sum_{\gamma \in \Gamma} \mathcal{N}(\gamma, e) \lambda(\gamma)-\mu(e)+\eta(e)\right)+\sum_{\gamma \in \Gamma} \lambda(\gamma) .
\end{aligned}
$$


As in the $p=1$ case, the Lagrangian is affine in the primal variables $t$ and $\rho$, so the dual objective function $\mathcal{F}_{\infty}(\lambda, \mu, \eta)$ is given by

$$
\mathcal{F}_{\infty}(\lambda, \mu, \eta):=\left\{\begin{array}{cc}
\sum_{\gamma \in \Gamma} \lambda(\gamma) & \text { if } \sum_{e \in E} \eta(e)=1 \text { and } \\
-\infty & \sum_{\gamma \in \Gamma} \mathcal{N}(\gamma, e) \lambda(\gamma)+\mu(e)-\eta(e)=0 \quad \forall e \in E,
\end{array}\right.
$$

Similarly to the case $p=1$, this produces the following form of the dual problem:

$$
\begin{aligned}
\text { maximize } & \sum_{\gamma \in \Gamma} \lambda(\gamma) \\
\text { subject to } & \sum_{\gamma \in \Gamma} \mathcal{N}(\gamma, e) \lambda(\gamma) \leq \eta(e) \quad \forall e \in E, \\
& \sum_{e \in E} \eta(e)=1, \\
& \lambda(\gamma) \geq 0 \quad \forall \gamma \in \Gamma .
\end{aligned}
$$

\section{Connections to COMmon Graph theoretic Quantities}

One of the key contributions of the present work is to make explicit the connections between the concept of $p$-modulus and the graph theoretic concepts of shortest path, effective conductance, and min-cut. The present section is devoted to establishing these connections.

4.1. Shortest path. The connection to shortest path is the broadest and simplest result to establish. Recall that $\ell(\Gamma)$, defined in Equation (11), is exactly the length of the shortest walk in $\Gamma$ (in terms of hops).

Theorem 4.1. Let $\Gamma$ be any family of walks, then

$$
\operatorname{Mod}_{\infty}(\Gamma)=\frac{1}{\ell(\Gamma)}
$$

Proof. Let $\gamma^{\prime} \in \Gamma$ be a walk such that $\ell\left(\gamma^{\prime}\right)=\ell(\Gamma)=\ell$. The density $\rho_{0} \equiv 1 / \ell$ is admissible, since for any $\gamma \in \Gamma$,

$$
\ell_{\rho_{0}}(\gamma)=\sum_{e \in E} \mathcal{N}(\gamma, e) \rho_{0}(e)=\frac{\ell(\gamma)}{\ell\left(\gamma^{\prime}\right)} \geq 1
$$

Thus $\operatorname{Mod}_{\infty}(\Gamma) \leq \ell^{-1}$. On the other hand, if $\rho \in A(\Gamma)$, then

$$
1 \leq \ell_{\rho}\left(\gamma^{\prime}\right)=\sum_{e \in E} \mathcal{N}\left(\gamma^{\prime}, e\right) \rho(e) \leq \ell\left(\gamma^{\prime}\right) \max _{e \in E} \rho(e)
$$

so every admissible density must satisfy $\mathcal{E}_{\infty}(\rho) \geq \ell^{-1}$.

4.2. Effective conductance. An undirected graph $G=(V, E, \sigma)$ is a model for a resistor network with edges representing resistors with conductances $\sigma$, connected at junctions represented by the vertices. Let $s$ and $t$ be distinct vertices in $V$. The effective conductance $C_{\text {eff }}(s, t)$ (the reciprocal of the effective resistance) between $s$ and $t$ can be found by minimizing the total power

$$
\text { Power }=\sum_{(x, y) \in E} \sigma(x, y)(\phi(x)-\phi(y))^{2}
$$


over all voltage potentials $\phi: V \rightarrow \mathbb{R}$ satisfying $\phi(s)=0$ and $\phi(t)=1$. The following theorem shows that, for $1<p<\infty$, the extremal density $\rho^{*}$ for the modulus of a connecting family of walks can be related to a generalized voltage potential. Such a result, in the language of extremal distance and in the special case $p=2$, first appeared in the work of Duffin [6] (see also [7, Proposition 6.2]). For a version in metric spaces, see [11, Theorem 7.31].

Theorem 4.2. Let $G=(V, E, \sigma)$ be an undirected graph, let $\Gamma=\Gamma(s, t)$ be the connecting family of walks from s to $t$, two distinct vertices in $V$, and let $1<p<\infty$. Let $\rho^{*}$ be the extremal density for Equation (3). Then there exists a vertex potential $\phi^{*}: V \rightarrow \mathbb{R}$ such that $\phi^{*}(s)=0, \phi^{*}(t)=1$, and

$$
\rho^{*}(x, y)=\left|\phi^{*}(x)-\phi^{*}(y)\right| \quad \forall(x, y) \in E .
$$

Moreover, this $\phi^{*}$ solves the optimization problem

$$
\begin{array}{ll}
\text { minimize } & \sum_{(x, y) \in E} \sigma(x, y)|\phi(x)-\phi(y)|^{p} \\
\text { subject to } & \phi(s)=0, \quad \phi(t)=1 .
\end{array}
$$

When $p=2$, it follows that $\operatorname{Mod}_{2}(\Gamma(s, t))=C_{\text {eff }}(s, t)$.

Proof. Let $\rho^{*} \in A(\Gamma)$ be the extremal density. Note that $\ell_{\rho^{*}}(\Gamma)=1$, for, if not, the density $\rho^{\prime}=\rho^{*} / \ell_{\rho^{*}}(\Gamma)$ is also admissible and has lower $p$-energy than $\rho^{*}$. Define $\phi^{*}(s)=0$ and for $x \in V \backslash\{s\}$,

$$
\phi^{*}(x)=\min _{\gamma \in \Gamma(s, x)} \ell_{\rho^{*}}(\gamma) .
$$

Since $\Gamma=\Gamma(s, t), \phi^{*}(t)=1$. To see that Equation (10) holds, define $\rho^{\prime}(x, y)=$ $\left|\phi^{*}(x)-\phi^{*}(y)\right|$ for $(x, y) \in E$. For any $\gamma=s v_{2} v_{3} \ldots v_{r} t \in \Gamma$,

$\ell_{\rho^{\prime}}(\gamma)=\left|\phi^{*}(s)-\phi^{*}\left(v_{2}\right)\right|+\left|\phi^{*}\left(v_{2}\right)-\phi^{*}\left(v_{3}\right)\right|+\cdots+\left|\phi^{*}\left(v_{r}\right)-\phi^{*}(t)\right| \geq\left|\phi^{*}(t)-\phi^{*}(s)\right|=1$, so $\rho^{\prime} \in A(\Gamma)$. Let $(x, y) \in E$ be an edge. Without loss of generality, $\phi^{*}(x) \leq \phi^{*}(y)$ and $y \neq s$. We claim that

$$
0 \leq \rho^{\prime}(x, y)=\phi^{*}(y)-\phi^{*}(x) \leq \rho^{*}(x, y) .
$$

The case $x=s$ is trivial. Suppose that $x \neq s$ and let $\gamma \in \Gamma(s, x)$ be a walk such that $\ell_{\rho^{*}}(\gamma)=\phi^{*}(x)$. Letting $\gamma^{\prime} \in \Gamma(s, y)$ be the walk obtained by first traversing $\gamma$ and then traversing the edge $(x, y)$, we have

$$
\phi^{*}(y) \leq \ell_{\rho^{*}}\left(\gamma^{\prime}\right)=\ell_{\rho^{*}}(\gamma)+\rho^{*}(x, y),
$$

which implies Equation (11).

Equation (11) implies that $\mathcal{E}_{p}\left(\rho^{\prime}\right) \leq \mathcal{E}_{p}\left(\rho^{*}\right)$ and, by uniqueness of the extremal density, $\rho^{*}=\rho^{\prime}$. To see that $\phi^{*}$ solves the optimization problem, let $\phi^{\prime}: V \rightarrow \mathbb{R}$ with $\phi^{\prime}(s)=0$ and $\phi^{\prime}(t)=1$ and define $\rho^{\prime}(x, y)=\left|\phi^{\prime}(x)-\phi^{\prime}(y)\right|$. As before, $\rho^{\prime} \in A(\Gamma)$ and so

$$
\sum_{(x, y) \in E} \sigma(x, y)\left|\phi^{*}(x)-\phi^{*}(y)\right|^{p}=\mathcal{E}_{p}\left(\rho^{*}\right) \leq \mathcal{E}_{p}\left(\rho^{\prime}\right)=\sum_{(x, y) \in E} \sigma(x, y)\left|\phi^{\prime}(x)-\phi^{\prime}(y)\right|^{p} .
$$


4.3. Max-Flow Min-Cut. Modulus is also closely related to the Max-Flow MinCut Theorem [8]. There are a number of different ways to see this; we shall focus on the connection to Ford and Fulkerson's original work. Let $G=(V, E, \sigma)$ be a weighted, undirected graph and let $s, t \in V$ be distinct vertices. Let $\Gamma$ be the family of simple paths from $s$ to $t$. $(\Gamma \subset \Gamma(s, t)$ is an essential subfamily in the sense of Theorem 3.1.) In the terminology of Ford and Fulkerson, the positive edge function $\sigma$ is called the capacity of the edge. A flow can be thought of as a function $\lambda: \Gamma \rightarrow[0, \infty)$ with the property that

$$
\sum_{\gamma \in \Gamma} \mathcal{N}(\gamma, e) \lambda(\gamma) \leq \sigma(e) \quad \forall e \in E
$$

and the value of such a flow the sum of $\lambda(\gamma)$ over all $\gamma \in \Gamma$. Thus, Ford and Fulkerson's maximal flow problem is equivalent to Equation (7) and forms a lower bound for the 1-modulus of $\Gamma$.

A disconnecting set is a subset of edges $D \subseteq E$ with the property that each $\gamma \in \Gamma$ traverses at least one edge in $D$, and a cut is a disconnecting set that contains no other disconnecting sets as proper subsets. The value of a disconnecting set $D$, denoted $v(D)$, is defined as the sum of the capacities of all edges in $D$. The MaxFlow Min-Cut Theorem is stated as follows.

Theorem 4.3 (Max-Flow Min-Cut). The values of the maximal flow and minimal cut on any graph are equal.

The connection between this and the 1-modulus is established in the following theorem.

Theorem 4.4. Let $G=(V, E, \sigma)$ be a weighted, undirected graph, let $s, t \in V$ be two distinct vertices and let $\Gamma(s, t)$ be the connecting family of walks between $s$ and t. Then $\operatorname{Mod}_{1}(\Gamma(s, t))$ is equal to the value of maximum flow (and minimum cut) from $s$ to $t$.

Proof. Let $\Gamma \subset \Gamma(s, t)$ be the family of simple paths connecting $s$ to $t$. $\Gamma$ is an essential subfamily and so $\operatorname{Mod}_{1}(\Gamma(s, t))=\operatorname{Mod}_{1}(\Gamma)$, which is bounded below by the value of the maximum flow, as we saw above.

Let $D \subseteq E$ be a disconnecting set of minimal value $v(D)$ and define the edge density $\rho$ as

$$
\rho(e):= \begin{cases}1 & \text { if } e \in D, \\ 0 & \text { otherwise }\end{cases}
$$

Since $D$ is a disconnecting set, $\rho \in A(\Gamma(s, t))$. Moreover,

$$
\operatorname{Mod}_{1}(\Gamma(s, t)) \leq \mathcal{E}_{1}(\rho)=\sum_{e \in E} \sigma(e) \rho(e)=\sum_{e \in D} \sigma(e)=v(D),
$$

providing the opposite inequality.

\section{Dependence on the parameter $p$}

The results of the preceding section are that the $p$-modulus can be thought of as a generalization of shortest path $(p=\infty)$, effective conductance $(p=2)$, and max-flow or min-cut $(p=1)$. Another way to see this is to consider an undirected, unweighted graph $G$ and let $\Gamma=\Gamma(s, t)$ be the connecting family of walks between distinct vertices $s$ and $t$. (Recall the example in Section 2.5) In this 
setting, $\operatorname{Mod}_{\infty}(\Gamma)$ equals the reciprocal of the length of the shortest path in $\Gamma$, while $\operatorname{Mod}_{1}(\Gamma)$ equals the maximum number of edge-disjoint paths from $s$ to $t$ (a consequence of Theorem 4.4 and Menger's theorem). $\operatorname{Mod}_{2}(\Gamma)$ strikes a balance between the other two, in a sense preferring both shortness and abundance in the collection of paths connecting $s$ to $t$.

In fact, as the present section demonstrates, the parameter $p$ can be thought of as a continuous "tuning parameter", which balances the sensitivity of the modulus between walk length and walk abundance. More precisely, given any graph $G=(V, E, \sigma)$ and any nonempty family of walks $\Gamma$, the function $p \mapsto \operatorname{Mod}_{p}(\Gamma)$ is continuous and decreasing. Moreover, $\operatorname{Mod}_{p}(\Gamma)^{1 / p}$ converges to $\operatorname{Mod}_{\infty}(\Gamma)$ as $p \rightarrow \infty$. In fact, with the correct normalization, the convergence is monotone (see Theorem 5.2).

We begin with bounds on the modulus.

Lemma 5.1. Let $G=(V, E, \sigma)$ be a graph with weights $\sigma>0$. Let the family $\Gamma$ and the parameter $1 \leq p<\infty$ be given. Then

$$
\frac{\sigma_{\min }}{\ell^{p}} \leq \operatorname{Mod}_{p}(\Gamma) \leq \frac{\sigma(E)}{\ell^{p}},
$$

where $\ell:=\ell(\Gamma)$,

$$
\sigma_{\min }:=\min _{e \in E} \sigma(e), \quad \text { and } \quad \sigma(E):=\sum_{e \in E} \sigma(e) .
$$

Proof. The upper bound can be obtained by considering the edge density $\rho \equiv \frac{1}{\ell}$. This density is admissible, because for all $\gamma \in \Gamma, \ell_{\rho}(\gamma)=\frac{1}{\ell} \ell(\gamma) \geq 1$. Thus

$$
\operatorname{Mod}_{p}(\Gamma) \leq \mathcal{E}_{p}(\rho)=\sum_{e \in E} \sigma(e) \frac{1}{\ell^{p}}=\frac{\sigma(E)}{\ell^{p}} .
$$

The lower bound can be obtained as follows. Let $\gamma^{\prime} \in \Gamma$ such that $\ell\left(\gamma^{\prime}\right)=$ $\ell(\Gamma)=\ell$. By Equation (9), for every $\rho \in A(\Gamma)$ there exists an edge $\tilde{e} \in E$ such that $\rho(\tilde{e}) \geq \ell^{-1}$. Thus,

$$
\mathcal{E}_{p}(\rho)=\sum_{e \in E} \sigma(e) \rho(e)^{p} \geq \sigma_{\min } \rho(\tilde{e})^{p} \geq \frac{\sigma_{\min }}{\ell^{p}} .
$$

Theorem 5.2. Let $G=(V, E, \sigma)$ and $\Gamma$ be given. Then

$$
\lim _{p \rightarrow \infty} \operatorname{Mod}_{p}(\Gamma)^{\frac{1}{p}}=\frac{1}{\ell(\Gamma)}=\operatorname{Mod}_{\infty}(\Gamma) .
$$

Moreover, the following monotonicity properties holds for $1 \leq p<q<\infty$ :

$$
\begin{aligned}
\operatorname{Mod}_{q}(\Gamma) & \leq \operatorname{Mod}_{p}(\Gamma), \\
\sigma(E)^{-1 / p} \operatorname{Mod}_{p}(\Gamma)^{1 / p} & \leq \sigma(E)^{-1 / q} \operatorname{Mod}_{q}(\Gamma)^{1 / q} .
\end{aligned}
$$

Remark 5.1. Observe that (12) and (14) combine to yield

$$
\sigma(E)^{-1 / p} \operatorname{Mod}_{p}(\Gamma)^{1 / p} \uparrow \operatorname{Mod}_{\infty}(\Gamma) \quad \text { as } p \rightarrow \infty .
$$

Proof. Taking the $p$ th root of the inequalities in Lemma 5.1 proves (12). Equation (13) follows from the fact that for any $\rho \in A^{*}(\Gamma)$ we have $0 \leq \rho \leq 1$, and since $q>p$, this implies that

$$
\mathcal{E}_{q}(\rho)=\sum_{e \in E} \sigma(e) \rho(e)^{q} \leq \sum_{e \in E} \sigma(e) \rho(e)^{p}=\mathcal{E}_{p}(\rho) .
$$


To prove monotonicity in (14) we use Hölder's inequality. Given $\rho \in A^{*}(\Gamma)$, assume that $1 \leq p<q<\infty$. Then, using the conjugate exponents $q / p$ and $q /(q-p)$,

$$
\begin{aligned}
\mathcal{E}_{p}(\rho) & =\sum_{e \in E} \sigma(e) \rho(e)^{p}=\sum_{e \in E} \sigma(e)^{p / q} \rho(e)^{p} \cdot \sigma(e)^{1-p / q} \\
& \leq\left(\sum_{e \in E} \sigma(e) \rho(e)^{q}\right)^{p / q}\left(\sum_{e \in E} \sigma(e)\right)^{1-p / q}=\sigma(E)^{1-p / q} \mathcal{E}_{q}(\rho)^{p / q}
\end{aligned}
$$

This can be rewritten as

$$
\sigma(E)^{-1 / p} \mathcal{E}_{p}(\rho)^{1 / p} \leq \sigma(E)^{-1 / q} \mathcal{E}_{q}(\rho)^{1 / q} .
$$

Picking $\rho$ to be extremal for $\operatorname{Mod}_{q}(\Gamma)$ we get (14).

Theorem 5.3. Let $G=(V, E, \sigma)$ and $\Gamma$ be given. Then the the function

$$
p \mapsto \operatorname{Mod}_{p}(\Gamma)
$$

is continuous for $1 \leq p<\infty$.

Proof. If we fix $p$ and let $q \downarrow p$, from (13) we get

$$
\limsup _{q \downarrow p} \operatorname{Mod}_{q}(\Gamma) \leq \operatorname{Mod}_{p}(\Gamma),
$$

and from (14) we get

$$
\liminf _{q \downarrow p} \operatorname{Mod}_{q}(\Gamma) \geq \liminf _{q \downarrow p} \sigma(E)^{1-q / p} \operatorname{Mod}_{p}(\Gamma)^{q / p}=\operatorname{Mod}_{p}(\Gamma) .
$$

So $\lim _{q \downarrow p} \operatorname{Mod}_{q}(\Gamma)=\operatorname{Mod}_{p}(\Gamma)$.

Likewise, if $q \uparrow p$, then from (13) we get

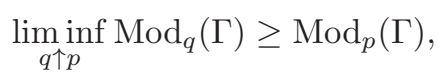

and from (14) we get

$$
\limsup _{q \uparrow p} \operatorname{Mod}_{q}(\Gamma) \leq \underset{q \uparrow p}{\limsup } \sigma(E)^{1-q / p} \operatorname{Mod}_{p}(\Gamma)^{q / p}=\operatorname{Mod}_{p}(\Gamma) .
$$

So $\lim _{q \rightarrow p} \operatorname{Mod}_{q}(\Gamma)=\operatorname{Mod}_{p}(\Gamma)$.

\section{Properties of extremal Densities}

We end with two properties of the extremal density in modulus problems: it is continuous as a function of $p$, and it is closely related to the gradient of the modulus with respect to the weights $\sigma$.

6.1. Continuity of the extremal density. This continuity result arises from Clarkson's inequalities. (See Theorem 2 of [5].) For a given $p \geq 1$, let $\|\cdot\|_{p}$ represent the standard $p$-norm on $\mathbb{R}^{m}$, defined on a density $\rho: E \rightarrow \mathbb{R}$ as

$$
\|\rho\|_{p}:=\left(\sum_{e \in E}|\rho(e)|^{p}\right)^{\frac{1}{p}} .
$$

In this context, the relevant Clarkson inequalities take the following form. 
Theorem 6.1. Let $p>1, p^{\prime}=p /(p-1), x, y \in \mathbb{R}^{m}$, and $\|\cdot\|_{p}$ be the standard p-norm.

If $p \geq 2$, then

$$
\left\|\frac{x+y}{2}\right\|_{p}^{p}+\left\|\frac{x-y}{2}\right\|_{p}^{p} \leq \frac{1}{2}\|x\|_{p}^{p}+\frac{1}{2}\|y\|_{p}^{p}
$$

If $1<p \leq 2$, then

$$
\left\|\frac{x+y}{2}\right\|_{p}^{p^{\prime}}+\left\|\frac{x-y}{2}\right\|_{p}^{p^{\prime}} \leq\left(\frac{1}{2}\|x\|_{p}^{p}+\frac{1}{2}\|y\|_{p}^{p}\right)^{\frac{p^{\prime}}{p}} .
$$

A first consequence of Clarkson's inequalities is that admissible densities that are almost-minimizers of the energy are close to the extremal density in $p$-norm.

Lemma 6.2. Let $G=(V, E, \sigma)$ be a graph with $0<\sigma_{\min } \leq \sigma$, and let $\Gamma$ be a family of walks on $G$. Assume $p>1$, and let $\rho^{*}$ be the unique extremal density for the $p$-modulus of $\Gamma$. Then, given any admissible density $\rho \in A(\Gamma)$ the following holds.

If $p \geq 2$, then

$$
\left\|\rho-\rho^{*}\right\|_{p}^{p} \leq \frac{2^{p-1}}{\sigma_{\min }}\left(\mathcal{E}_{p}(\rho)-\operatorname{Mod}_{p}(\Gamma)\right) .
$$

If $1<p \leq 2$, then

$$
\left\|\rho-\rho^{*}\right\|_{p}^{p^{\prime}} \leq\left(\frac{2^{p}}{\sigma_{\min }}\right)^{\frac{p^{\prime}}{p}}\left[\left(\operatorname{Mod}_{p}(\Gamma)+\frac{1}{2}\left(\mathcal{E}_{p}(\rho)-\operatorname{Mod}_{p}(\Gamma)\right)\right)^{\frac{p^{\prime}}{p}}-\operatorname{Mod}_{p}(\Gamma)^{\frac{p^{\prime}}{p}}\right] .
$$

Proof. It is convenient to define two additional edge densities, $f$ and $f^{*}$, as

$$
f(e):=\sigma(e)^{\frac{1}{p}} \rho(e) \quad \text { and } \quad f^{*}(e):=\sigma(e)^{\frac{1}{p}} \rho^{*}(e) .
$$

Then

$$
\|f\|_{p}^{p}=\mathcal{E}_{p}(\rho) \quad \text { and } \quad\left\|f^{*}\right\|_{p}^{p}=\mathcal{E}_{p}\left(\rho^{*}\right)=\operatorname{Mod}_{p}(\Gamma)
$$

and

$$
\left\|\frac{f-f^{*}}{2}\right\|_{p}^{p}=\frac{1}{2^{p}} \sum_{e \in E} \sigma(e)\left|\rho(e)-\rho^{*}(e)\right|^{p} \geq \frac{\sigma_{\min }}{2^{p}}\left\|\rho-\rho^{*}\right\|_{p}^{p} .
$$

Also,

$$
\left\|\frac{f+f^{*}}{2}\right\|_{p}^{p}=\sum_{e \in E} \sigma(e)\left|\frac{\rho(e)+\rho^{*}(e)}{2}\right|^{p}=\mathcal{E}_{p}\left(\frac{\rho(e)+\rho^{*}(e)}{2}\right) \geq \operatorname{Mod}_{p}(\Gamma),
$$

where the final inequality follows from the fact that the admissible set $A(\Gamma)$ is convex, so that $\left(\rho+\rho^{*}\right) / 2 \in A(\Gamma)$.

When $p \geq 2$, Equations (21) through (23) combined with Theorem6.1 show that

$$
\frac{\sigma_{\min }}{2^{p}}\left\|\rho-\rho^{*}\right\|_{p}^{p} \leq \frac{1}{2} \mathcal{E}_{p}(\rho)+\frac{1}{2} \operatorname{Mod}_{p}(\Gamma)-\operatorname{Mod}_{p}(\Gamma),
$$


which proves (19). When $1<p \leq 2$, a similar argument shows that

$$
\left(\frac{\sigma_{\min }}{2^{p}}\right)^{\frac{p^{\prime}}{p}}\left\|\rho-\rho^{*}\right\|_{p}^{p^{\prime}} \leq\left(\frac{1}{2} \mathcal{E}_{p}(\rho)+\frac{1}{2} \operatorname{Mod}_{p}(\Gamma)\right)^{\frac{p^{\prime}}{p}}-\operatorname{Mod}_{p}(\Gamma)^{\frac{p^{\prime}}{p}}
$$

implying (20).

Theorem 6.3. Let $\Gamma$ be a family of walks on a graph $G$ and let $1<p<\infty$. Then

$$
\lim _{q \rightarrow p}\left\|\rho_{p}-\rho_{q}\right\|=0,
$$

where $\rho_{p}$ and $\rho_{q}$ are the unique extremal densities for $p$ and $q>1$ respectively.

Note that the norm in the theorem is irrelevant, since all norms on $\mathbb{R}^{m}$ are equivalent.

Proof. By Lemma 6.2, it is enough to show that $\mathcal{E}_{p}\left(\rho_{q}\right) \rightarrow \operatorname{Mod}_{p}(\Gamma)$ as $q \rightarrow p$. For $q>p$, Equation (17) shows that

$$
\operatorname{Mod}_{p}(\Gamma) \leq \mathcal{E}_{p}\left(\rho_{q}\right) \leq \sigma(E)^{1-\frac{p}{q}} \mathcal{E}_{q}\left(\rho_{q}\right)^{\frac{p}{q}}=\sigma(E)^{1-\frac{p}{q}} \operatorname{Mod}_{q}(\Gamma)^{\frac{p}{q}}
$$

and for $q<p$, Equation (16) shows that

$$
\operatorname{Mod}_{p}(\Gamma) \leq \mathcal{E}_{p}\left(\rho_{q}\right) \leq \mathcal{E}_{q}\left(\rho_{q}\right)=\operatorname{Mod}_{q}(\Gamma) .
$$

Then, by continuity (Theorem 5.3), letting $q$ tend to $p$ yields the result.

6.2. Extremal density as a gradient. We now turn our attention to the behavior of the modulus as a function of the weights $\sigma: E \rightarrow(0, \infty)$. For this discussion, we fix a graph $G=(V, E)$ and a nonempty family of walks $\Gamma$, but allow the edge weights, $\sigma$, to vary. In order to make explicit the dependence on the weights $\sigma$, we use the notation $\mathcal{E}_{p}(\rho ; \sigma)$ and $\operatorname{Mod}_{p}(\Gamma ; \sigma)$ to denote the $p$-energy of $\rho$ and the $p$-modulus of $\Gamma$ respectively. We restrict attention to $p<\infty$, since $\mathcal{E}_{\infty}$ and $\operatorname{Mod}_{\infty}$ do not depend on $\sigma$. The next two lemmas show that modulus is continuous and concave in $\sigma$.

Lemma 6.4. Let $1 \leq p<\infty$. The function $\sigma \mapsto \operatorname{Mod}_{p}(\Gamma ; \sigma)$ is Lipschitz continuous.

Proof. Let $\sigma_{1}, \sigma_{2}: E \rightarrow(0, \infty)$ and let $\rho_{1}$ and $\rho_{2}$ be extremal densities for $\operatorname{Mod}_{p}\left(\Gamma ; \sigma_{1}\right)$ and $\operatorname{Mod}_{p}\left(\Gamma ; \sigma_{2}\right)$ respectively. Without loss of generality, assume that $\operatorname{Mod}_{p}\left(\Gamma ; \sigma_{1}\right) \leq \operatorname{Mod}_{p}\left(\Gamma ; \sigma_{2}\right)$. From Lemma 2.2, it follows that $\left|\rho_{1}\right| \leq 1$, so

$$
\begin{aligned}
& \operatorname{Mod}_{p}\left(\Gamma ; \sigma_{2}\right)-\operatorname{Mod}_{p}\left(\Gamma ; \sigma_{1}\right) \leq \mathcal{E}_{p}\left(\rho_{1} ; \sigma_{2}\right)-\mathcal{E}_{p}\left(\rho_{1} ; \sigma_{1}\right) \\
& =\sum_{e \in E} \sigma_{2}(e) \rho_{1}(e)^{p}-\sum_{e \in E} \sigma_{1}(e) \rho_{1}(e)^{p} \\
& =\sum_{e \in E}\left(\sigma_{2}(e)-\sigma_{1}(e)\right) \rho_{1}(e)^{p} \leq \sum_{e \in E}\left|\sigma_{2}(e)-\sigma_{1}(e)\right| \leq m\left\|\sigma_{2}-\sigma_{1}\right\|_{\infty},
\end{aligned}
$$

where $\|\cdot\|_{\infty}$ is the standard max norm on $\mathbb{R}^{m}$.

Lemma 6.5. Let $1 \leq p<\infty$. The function $\sigma \mapsto \operatorname{Mod}_{p}(\Gamma ; \sigma)$ is concave. 
Proof. Let $\sigma_{1}, \sigma_{2}: E \rightarrow(0, \infty)$ and let $\theta \in[0,1]$. Let $\rho_{1}, \rho_{2}$ and $\rho^{*}$ be extremal densities for $\sigma_{1}, \sigma_{2}$ and $\sigma^{*}:=\theta \sigma_{1}+(1-\theta) \sigma_{2}$, respectively. Then

$$
\begin{aligned}
\theta \operatorname{Mod}_{p}\left(\Gamma ; \sigma_{1}\right)+(1-\theta) \operatorname{Mod}_{p}\left(\Gamma ; \sigma_{2}\right) & \leq \theta \sum_{e \in E} \sigma_{1}(e) \rho^{*}(e)^{p}+(1-\theta) \sum_{e \in E} \sigma_{2}(e) \rho^{*}(e)^{p} \\
& =\sum_{e \in E} \sigma^{*}(e) \rho^{*}(e)^{p}=\operatorname{Mod}_{p}\left(\Gamma ; \theta \sigma_{1}+(1-\theta) \sigma_{2}\right) .
\end{aligned}
$$

For $p>1$, the following theorem provides an interpretation of the unique extremal density in terms of the gradient of $\operatorname{Mod}_{p}(\Gamma ; \sigma)$ with respect to $\sigma$.

Theorem 6.6. Let $1<p<\infty$ and define the function $F(\sigma):=\operatorname{Mod}_{p}(\Gamma ; \sigma)$ on weights $\sigma: E \rightarrow(0, \infty)$. Given a weight $\sigma$, let $\rho_{\sigma}^{*}$ denote the unique extremal density for $\operatorname{Mod}_{p}(\Gamma ; \sigma)$. Then

$$
\frac{\partial F(\sigma)}{\partial \sigma(e)}=\rho_{\sigma}^{*}(e)^{p}
$$

Proof. Let $\sigma: E \rightarrow(0, \infty)$ and $\eta: E \rightarrow \mathbb{R}$. For $h>0$ sufficiently small, $\sigma_{h}:=\sigma+h \eta$ is positive. Let $\rho_{h}^{*}$ be the extremal density for $\operatorname{Mod}_{p}\left(\Gamma ; \sigma_{h}\right)$, and let $\rho^{*}=\rho_{0}^{*}$, the extremal density for $\operatorname{Mod}_{p}(\Gamma ; \sigma)$. Note that, for any $\rho: E \rightarrow \mathbb{R}$,

$$
\mathcal{E}_{p}\left(\rho ; \sigma_{h}\right)=\sum_{e \in E}(\sigma(e)+h \eta(e))|\rho(e)|^{p}=\mathcal{E}_{p}(\rho ; \sigma)+h \sum_{e \in E} \eta(e)|\rho(e)|^{p} .
$$

In particular,

$$
\begin{aligned}
\operatorname{Mod}_{p}\left(\Gamma ; \sigma_{h}\right) & \leq \mathcal{E}_{p}\left(\rho^{*} ; \sigma_{h}\right)=\mathcal{E}_{p}\left(\rho^{*} ; \sigma\right)+h \sum_{e \in E} \eta(e) \rho^{*}(e)^{p} \\
& =\operatorname{Mod}_{p}(\Gamma ; \sigma)+h \sum_{e \in E} \eta(e) \rho^{*}(e)^{p}
\end{aligned}
$$

and

$$
\begin{aligned}
\operatorname{Mod}_{p}\left(\Gamma ; \sigma_{h}\right) & =\mathcal{E}_{p}\left(\rho_{h}^{*} ; \sigma_{h}\right)=\mathcal{E}_{p}\left(\rho_{h}^{*} ; \sigma\right)+h \sum_{e \in E} \eta(e) \rho_{h}^{*}(e)^{p} \\
& \geq \operatorname{Mod}_{p}(\Gamma ; \sigma)+h \sum_{e \in E} \eta(e) \rho_{h}^{*}(e)^{p}
\end{aligned}
$$

Combined, these two inequalities imply that for $h>0$ sufficiently small

$$
\sum_{e \in E} \eta(e) \rho_{h}^{*}(e)^{p} \leq \frac{F(\sigma+h \eta)-F(\sigma)}{h} \leq \sum_{e \in E} \eta(e) \rho^{*}(e)^{p} .
$$

Now, since each $\rho_{h}^{*} \in A^{*}(\Gamma)$ by Lemma 2.2 .

$$
\begin{aligned}
\mathcal{E}_{p}\left(\rho_{h}^{*} ; \sigma\right)-\operatorname{Mod}_{p}(\Gamma ; \sigma) & =\mathcal{E}_{p}\left(\rho_{h}^{*} ; \sigma_{h}\right)-h \sum_{e \in E} \eta(e) \rho_{h}^{*}(e)^{p}-\operatorname{Mod}_{p}(\Gamma ; \sigma) \\
& \leq\left|\operatorname{Mod}_{p}\left(\Gamma ; \sigma_{h}\right)-\operatorname{Mod}_{p}(\Gamma ; \sigma)\right|+h \sum_{e \in E}|\eta(e)| .
\end{aligned}
$$

Lemma 6.4 implies that the right-hand side vanishes in the limit as $h \rightarrow 0$, so $\mathcal{E}_{p}\left(\rho_{h}^{*} ; \sigma\right) \rightarrow \operatorname{Mod}_{p}(\Gamma ; \sigma)$, and Lemma 6.2 then implies that $\rho_{h}^{*} \rightarrow \rho^{*}$. Taking the 
limit in (25) yields

$$
\lim _{h \rightarrow 0^{+}} \frac{F(\sigma+h \eta)-F(\sigma)}{h}=\sum_{e \in E} \eta(e) \rho^{*}(e)^{p},
$$

which, since $\eta$ was arbitrary, implies (24).

\section{Related WORK}

The concept of the modulus of a family of walks on a graph is a natural analog of the continuum definition of modulus of a family of curves in the plane, introduced by Beurling [4 and Ahlfors [1. The task of detailing its historical development is complicated by the facts that modulus seems almost infinitely generalizable and that similar ideas exist in different fields, with different notation and terminology. Certainly, the work on maximal flow by Ford and Fulkerson [8] is closely related to modulus (see Theorem 4.4), as is the work on extremal length in networks by Duffin [6]. Indeed, Duffin's paper cites Beurling and Ahlfors as well as Ford and Fulkerson in the introduction, and Duffin's extremal width is equivalent to a particular modulus problem. (Compare Theorem 2 of [6] to Theorem 4.2 of the present work.) More recently, one finds the work of Schramm 14, which uses a form of modulus to study square tilings, and the work of Haïssinsky [10, which develops a discrete version of the modulus quite similar to the definition in the present work, but with a different goal in mind.

\section{Discussion}

This paper presents an exploration, in a fairly general setting, of the ways in which modulus generalizes and expands upon important graph theoretical concepts. Modulus is shown to generalize three fundamental concepts from graph theoryshortest path, effective conductance, and min-cut - and to interpolate, in a sense, among these three concepts. These results depend upon lower and upper bounds on the modulus, which are established using tools from convex optimization.

With these connections firmly in place, a number of questions naturally arise. For example, resistance distance has proven quite valuable in a wide variety of applications. As we have shown, on a weighted, undirected graph, this distance is equivalent to the 2-modulus, in the sense that the resistance distance between $s$ and $t$ in $V$ is $\operatorname{Mod}_{2}(\Gamma(x, y))^{-1}$. Is $p=2$ the only useful case? Or are there applications for which some other value of $p$ is useful? Moreover, there is no obvious sense of effective resistance on directed graphs, but such a concept may well be useful (see, e.g., [15].) We are aware, for example, of a recent arXiv paper [16] which constructs a notion of resistance distance on directed graphs that preserves certain important control theoretic properties. The average of $\operatorname{Mod}_{p}(\Gamma(x, y))$ and $\operatorname{Mod}_{p}(\Gamma(y, x)) \operatorname{might}$ also be an interesting quantity to look at in applications.

There are also several interesting open questions regarding the dependence of the modulus and extremal density on $p$ beyond the continuity and monotonicity proved in Section 5. For example, it is not yet clear how smooth the function $p \mapsto \operatorname{Mod}_{p}(\Gamma)$ is in general. Moreover, it would be interesting to better understand the behavior of the unique extremal density $\rho_{p}$ either as $p \rightarrow 1$ or as $p \rightarrow \infty$. In both cases, one might wonder if there is a limiting density and, if so, if the limiting density is extremal for the 1- or $\infty$-modulus. 
Another potentially interesting direction is graph optimization, similar in spirit to the results of Reference [9]. As shown in Section 6.2, the function $\sigma \mapsto \operatorname{Mod}_{p}(\Gamma ; \sigma)$ is concave, so the problem of maximizing the modulus subject to convex constraints on $\sigma$ (e.g., that the sum of $\sigma$ over all edges is bounded) is a convex optimization problem whose solution could give some insight into the structure of a graph relative to a given family of walks.

As mentioned in the previous section, generalizations of the modulus presented here seem almost endless. Some obvious directions of research include the replacement of edge densities with vertex densities, or using a mixture of both. Moreover, the objects in $\Gamma$ do not actually need to be walks. One might consider a "cut modulus", for example, where $\Gamma$ is the family of all edge cuts separating two given sets of vertices.

Another interesting line of inquiry lies in understanding what the modulus can say about the structure of a graph. As mentioned in the introduction, for example, it can be interesting to consider moduli of families of walks from $s$ to $t$ passing through $u$. One might also consider other families, such as the family of simple paths with at least $L$ hops, or the family of simple cycles with diameter at least $D$. What might the moduli of these families say about the structure of the graph? What is the expected modulus of these families on a given random class of graph?

Perhaps even more intriguing is the interpretation offered by the dual formulation of Section 3 . In the dual interpretation, the edge density $\rho$ is replaced by nonnegative function $\lambda$ on $\Gamma$. Through duality, $\lambda$ is related to the sensitivity of the modulus to the admissibility constraints, giving a natural ranking of the importance of the walks in $\Gamma$. Those walks with larger $\lambda$ are more important in the modulus problem than those with smaller $\lambda$. This perspective of most important walks is rich for exploration and has been developed in [2]. For instance, by replacing $\Gamma$ by some properly chosen subset $\Gamma^{\prime}$ with large $\lambda$ values, one can obtain a subfamily with nearly the same modulus as $\Gamma$. This viewpoint also lends itself to a concept of sparsification subordinate to a modulus problem: by choosing a subfamily $\Gamma^{\prime}$ of most important walks, and then removing from $E$ all edges that do not intersect $\Gamma^{\prime}$, it is possible to produce a subgraph that approximates the behavior of the original graph from the perspective of the given modulus problem.

\section{ACKNOWLEDGMEnTS}

The authors are grateful to Professors Marianne Korten and David Yetter for organizing an exceptional summer research program. We also thank the anonymous referee for a very careful reading and helpful suggestions that improved this paper.

\section{REFERENCES}

[1] L. V. Ahlfors, Collected papers. Vol. 1, Contemporary Mathematicians, Birkhäuser, Boston, Mass., 1982. 1929-1955; Edited with the assistance of Rae Michael Shortt. MR688648 (84k:01066a)

[2] N. Albin and P. Poggi-Corradini, The dual method for p-modulus on graphs. Preprint.

[3] N. Albin and P. Poggi-Corradini, F. Darabi Sahneh, and M. Goering, Modulus of families of walks on graphs. arXiv:1401.7640 (http://arxiv.org/abs/1403.5750).

[4] A. Beurling, The collected works of Arne Beurling. Vol. 1, Contemporary Mathematicians, Birkhäuser Boston, Inc., Boston, MA, 1989. Complex analysis; Edited by L. Carleson, P. Malliavin, J. Neuberger and J. Wermer. MR1057613(92k:01046a)

[5] J. A. Clarkson, Uniformly convex spaces, Trans. Amer. Math. Soc. 40 (1936), no. 3, 396-414, DOI 10.2307/1989630. MR1501880 
[6] R. J. Duffin, The extremal length of a network, J. Math. Anal. Appl. 5 (1962), 200-215. MR0143468(26 \#1024)

[7] J. Ericson, P. Poggi-Corradini, and H. Zhang, Effective resistance on graphs and the epidemic quasimetric, Involve 7 (2014), no. 1, 97-124, DOI 10.2140/involve.2014.7.97. MR3127324

[8] L. R. Ford Jr. and D. R. Fulkerson, Maximal flow through a network, Canad. J. Math. 8 (1956), 399-404. MR0079251 (18,56h)

[9] A. Ghosh, S. Boyd, and A. Saberi, Minimizing effective resistance of a graph, SIAM Rev. 50 (2008), no. 1, 37-66, DOI 10.1137/050645452. MR2403057 (2009j:05071)

[10] P. Haïssinsky, Empilements de cercles et modules combinatoires (French, with English and French summaries), Ann. Inst. Fourier (Grenoble) 59 (2009), no. 6, 2175-2222. MR2640918 (2011d:52043)

[11] J. Heinonen, Lectures on analysis on metric spaces, Universitext, Springer-Verlag, New York, 2001. MR1800917 (2002c:30028)

[12] D. J. Klein and M. Randić, Resistance distance, J. Math. Chem. 12 (1993), no. 1-4, 8195, DOI 10.1007/BF01164627. Applied graph theory and discrete mathematics in chemistry (Saskatoon, SK, 1991). MR1219566 (94d:94041)

[13] R. T. Rockafellar, Convex analysis, Princeton Mathematical Series, No. 28, Princeton University Press, Princeton, N.J., 1970. MR0274683 (43 \#445)

[14] O. Schramm, Square tilings with prescribed combinatorics, Israel J. Math. 84 (1993), no. 1-2, 97-118, DOI 10.1007/BF02761693. MR1244661 (94h:52045)

[15] H. Shakeri, P. Poggi-Corradini, C. Scoglio, and N. Albin, Generalized network measures based on modulus of families of walks. Preprint.

[16] G. F. Young, L. Scardovi, and N. E. Leonard, A new notion of effective resistance for directed graphs-Part I: Definition and properties. http://arxiv.org/abs/1310.5163.

Department of Mathematics, Kansas State University, 138 Cardwell Hall, ManhatTAN, KANSAS 66506

E-mail address: albin@math.ksu.edu; pietro@math.ksu.edu 